\title{
NEW CONTROL SYSTEM OF THE INSERTION DEVICES AT THE KEK PHOTON FACTORY STORAGE RING
}

\author{
T. Shioya and $\underline{K}$. Tsuchiya ${ }^{\#}$ \\ KEK PF, Oho 1-1, Tsukuba, Ibaraki 305, Japan
}

\section{Abstract}

The control system of the insertion devices (IDs) at the Photon Factory storage ring ( $\mathrm{PF}$ ring) has been renewed in accordance with the high-brilliance project of the PF ring. It consists of a workstation and VME systems, which are linked to dedicated user PCs in the SR experimental hall. Each user of the IDs can change the magnetic gap independently by accessing this workstation during their SR experiment. The closed-orbit affected by changing the gap is corrected using correction magnets system located at both ends of each ID. The vertical fluctuation of the gradients of the photon beam axis is suppressed to less than $2 \mu \mathrm{rad}$.

\section{INTRODUCTION}

In the Photon Factory storage ring (PF ring; $2.5 \mathrm{GeV}$ ), five permanent-magnet insertion devices (IDs) are in operation, namely Undulator U\#02; multi-pole wiggler MPW\#13,16; revolver Rev\#19; and elliptical multi-pole wiggler EMPW\#28. Table.1 lists their main parameters. Each ID had been controlled by an individual personal computer (ID-PC) to change the magnetic gap and to correct the closed-orbit distortion (COD). The ID-PCs had been placed beside a ring control room. Users of IDs had called up a ring operator to change the gap for choosing the photon energy during their SR experiments.

Table 1: Main parameters of the IDs

\begin{tabular}{|c|c|c|c|c|}
\hline Name & $\begin{array}{c}\text { operation } \\
\text { mode }\end{array}$ & $\begin{array}{c}\boldsymbol{\lambda} \mathbf{u} \\
(\mathbf{c m})\end{array}$ & $\mathbf{N}$ & Kmax \\
\hline U\#02 & & 6 & 60 & 2.3 \\
\hline MPW\#13 & & 18 & 13 & 25 \\
\hline MPW\#16 & & 12 & 26 & 16.8 \\
\hline \multirow{2}{*}{ Rev\#19 } & $\mathrm{A}$ & 5 & 46 & 1.3 \\
\cline { 2 - 5 } & $\mathrm{B}$ & 7.2 & 32 & 2.7 \\
\cline { 2 - 5 } & $\mathrm{C}$ & 10 & 23 & 5 \\
\cline { 2 - 5 } & $\mathrm{D}$ & 16.4 & 14 & 9.5 \\
\hline \multirow{2}{*}{$\begin{array}{c}\text { EMPW } \\
\text { \#28 }\end{array}$} & $\begin{array}{c}\text { elliptical } \\
\text { MPW }\end{array}$ & 16 & 21 & $\begin{array}{c}\mathrm{Kx}: 0.3 \\
\mathrm{Ky}: 15\end{array}$ \\
\cline { 2 - 5 } & helical U & & & $(\mathrm{Kx}=\mathrm{Ky})$ \\
\cline { 2 - 5 } & linear U & & & \\
\hline
\end{tabular}

"Email: tsuchiya@mail.kek.jp
This control system of the IDs has been renewed in accordance with reconstruction for the brillianceupgrading project of the PF ring [1]. This new control system has one workstation for the IDs (ID-WS) and five VME systems. Each of the VME systems performs the same role as that of the ID-PC. It controls both the gapcontroller of the ID and the steering magnets system for the COD correction, using the GPIB interface. The IDWS controls these VME systems and communicates with them through the local-area network (LAN). Figure 1 shows a schematic view of the new control system of the IDs. A terminal for the man-machine interface (MMI) placed in the ring control room displays all status of the IDs at every moment. From this MMI terminal, the ring operator can control all IDs and steering magnets directly sending requests to the ID-WS.

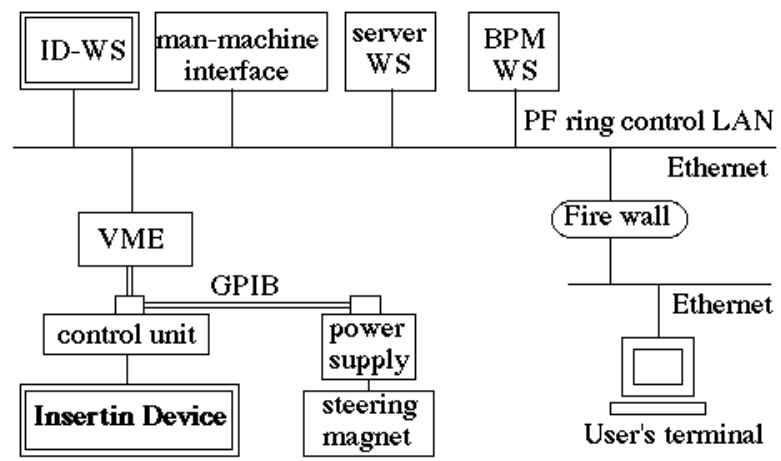

Figure 1: Schematic view of the new control system of the insertion devices.

Each beam line of the IDs has a dedicated PC terminal, which has permission to access the ID-WS. Users can send requests to the ID-WS for changing the gap through network communication. When the ID-WS has received a service request, it judges the condition for changing the gap by checking the operation status of the PF ring. In the case of the enabled condition, the VME system executes the command from the ID-WS. After changing the gap, the result is reported to the user's PC and the MMI. Also, information concerning the present gaps of every ID is open to the public on a Web page [2]. The logging data for gap changes are saved in a database of the PF ring.

This new control system of the IDs had been developed in the MPW\#13 as the first case. Operation of the new control system in the MPW\#13 was started in February, 1998. The control systems for the U\#02 and EMPW\#28 
were unified to the new control system in October, 1998. The remaining systems, for the MPW\#16 and Rev\#19, will be unified in March, 1999. The system for Rev\#19 is being developed by the Institute of Materials Structure, University of Tokyo.

\section{THE COD CORRECTION FOR THE IDS}

\subsection{System}

For the users of IDs, it is important to vary the photon energy freely by changing the gap during their SR experiments. This is the meaning of independent tuning of the IDs. However, a closed-orbit is sometime affected by changing the gap, which causes a fluctuation of the photon-beam axis at all beam-lines. To suppress this fluctuation by changing the gap, a COD correction has been made simultaneously by the steering magnets system located at both ends of each ID. Each of the VME systems has a corrective current data table for the steering magnets over the entire operating range of the gap. These current data tables for the steering magnets of all IDs have been taken for independent tuning before the user operation. The VME reads the present gap and sets a suitable current repetitively for changing the gap. The typical speed of changing the gap of ID is $20 \mathrm{~mm} / \mathrm{min}$, and the correction frequency is $7 \mathrm{~Hz}$ in the case of MPW\#13.

The steering magnet can generate an integral magnetic field up to 2000 Gauss-cm either in the horizontal or vertical direction, coupled with a 12-bits DC power supply (HP6633A). A permalloy has been adopted as the core material of the magnet in order to reduce the remnant magnetic field as much as possible and to obtain reliable reproducibility.

\subsection{Acquisition of corrective data}

The PF ring has several operation modes besides the nominal $2.5 \mathrm{GeV}$ operation, such as $3 \mathrm{GeV}$ operation. When the orbit of the electron beam is changed according to the operation mode, all correction data tables of the IDs are refreshed before user SR experiments in order to satisfy the condition for independent tuning of the IDs. The new data-acquisition program in the ID-WS searches these corrective current data so as to fit the closed-orbit to the standard one by the least mean-squares method over the operating range of the gap step by step. To obtain information about the beam position, a program to collect corrective data can use the new beam-position monitor (BPM) system of the PF ring [3] or the photon-BPM (PBPM) in the beam line [4].

The system of the BPMs was also renovated accompanying reconstruction of the PF ring. The number of new BPMs is 65 and the frequency of the COD measurement is $50 \mathrm{~Hz}$. The relative accuracy of the measured beam position is less than $5 \mu \mathrm{m}$. The averaged data of the BPMs during one second have been sent to the data-storage area, the so-called Data channel (DCh), in the shared memory of the server workstation [5]. The IDWS can read the BPMs data accessing to the DCh, and calculates the optimum corrective current both the horizontal or vertical direction. To search for vertical corrective current data, four PBPMs at the beam lines (BL04, 06, 12, 21) are available.

\subsection{Results of a COD correction}

The corrective current data for the present closed orbit with an emittance of $36 \mathrm{~nm}$-rad was adjusted using the new BPM system for both the vertical and horizontal directions. During the measurements, development of the new control system for the IDs was underway. Thus, for acquiring corrective data, the program of the old control system for the IDs was used, which was modified so as to use the new BPM data in the DCh. The program iterated the correction of the beam position at the selected three BPMs until the differences from the standard position had been reduced to less than $10 \mu \mathrm{m}$. Figure 2 shows typical results of the COD correction during the change in the gap. The examples are for the case of U\#02 and MPW\#13. (a) U\#02 horizontal

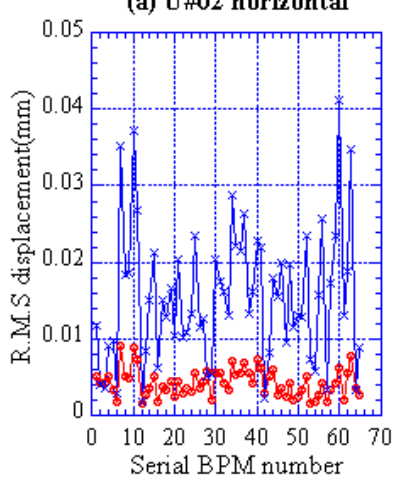

(c) MPW\#13 horizontal

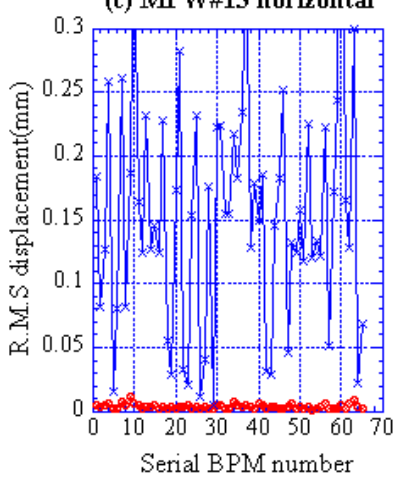

(b) U\#02 vertical

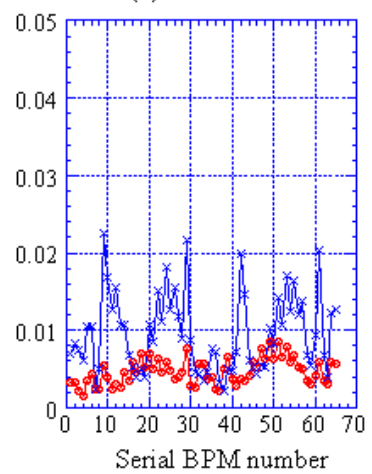

(d) MPW\#13 vertical

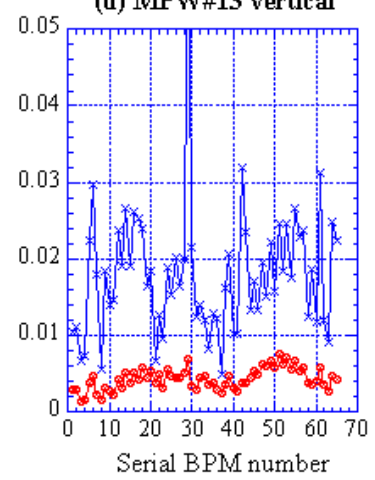

Figure 2: The results of the COD correction for the IDs. (circles: correction ON, crosses: correction OFF) 
The horizontal and vertical r.m.s. displacements at the BPMs are plotted during a change from the minimum gap to the maximum gap. The displacements after a correction have been reduced to less than $10 \mu \mathrm{m}$ all around the ring in the both directions. The results are the same in the cases of the other IDs. Because the time needed to measure one of these data is about 10 minutes, which is limited by the speed of changing the gap, these data of the displacements involve both the drift of the electron beam and the fluctuation due to the errors of the BPMs. Figure 3 shows a comparison between the measured displacements with a correction and the background drift in the vertical direction. The COD corrections for the gap-changing work to suppress the fluctuation of the closed-orbit within the twice comparisons with that of the orbit in the steady state.

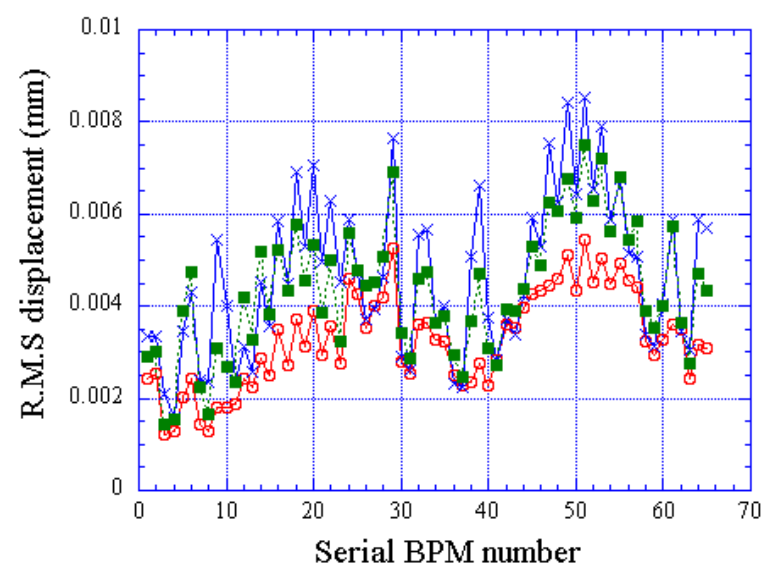

Figure 3: The comparisons of the vertical fluctuations (r.m.s) of the orbit. (circles: the background drift, crosses: the case of U\#02, squares: the case of MPW\#13)

The reliability of the corrective data was checked using four PBPMs located in the beam lines: BL04, 06, 12 and 21 , respectively. The fluctuation data in the vertical SR beam positions of the PBPMs was recorded using chart recorders. Most of the fluctuations remained within the range of $20 \mu \mathrm{m}$ from the standard position, which corresponds to an axial gradient of $2 \mu \mathrm{rad}$. A change in the gap beyond this range is allowed only just after the injection, so as to change the operation mode of the Rev\#19 or the EMPW\#28. The conformation of the corrective data using PBPMs is always performed after a long shutdown of the PF ring.

In practical operation of the PF ring for user experiments, the fast $(10 \mathrm{~Hz})$ global feedback system works in order to stabilize the fluctuation of the vertical orbit [6]. Thus, the vertical orbit fluctuation during a change in the gap should be more reduced by using the feedback system simultaneously during the period of user experiments.

\subsection{Improvement}

The average time to take one corrective data set was about 2 hours. It was reduced to half compared with the case of using the old BPM system. However, the total time required to take data for all IDs was still several days. A curtailment of the time for data-acquisition is one of the important subjects for the new control system. A test of the new program in the ID-WS to collect corrective data was performed well and efficiently at MPW\#13 using the new BPM system for both the vertical and horizontal directions. The new corrective data-acquisition system will be commissioned in April, 1999.

On the other hand, 12 new PBMPs were installed in the $\mathrm{PF}$ ring by the SR monitor group. Commissioning of the new PBPM systems is underway. The ID-WS communicates to the workstation for the new PBPM systems in order to obtain more information about the vertical SR positions. To utilize the additional new PBPMs systems, we will improve taking corrective data and checking it more carefully.

\section{SUMMARY}

The new control system for the IDs at the PF ring has been developed and operation of the system has started. The fluctuation of the electron beam-position due to changing the gap is suppressed to less than $10 \mu \mathrm{m}$ for independent tuning of the IDs.

\section{REFERENCES}

[1] M. Katoh, Y. Hori, Y. Kobayashi, S. Sakanaka, M. Izawa, K. Haga, T. Honda, T. Mitsuhashi, T. Obina, C. O. Pak, K. Ohmi, K. Tsuchiya, N. Kanaya, A. Ueda, M. Tadano, Y. Takiyama, S. Asaoka, T. Nogami, T. Shioya, A. Mishina, Y. Sato, S. Tokumoto, T. Takahashi, T. Kasuga, H. Maezawa, M. Kobayashi, and H. Kobayakawa, J. Synchrotron Rad. (1998). 5, 366

[2] http://pfrpc1.kek.jp/nogami/DchJava/MPW.html

[3] K. Haga, J. Synchrotron Rad. (1998). 5, 624

[4] T. Mitsuhashi, K. Haga, and T. Katsura, IEEE PAC, Washington (1987), 576

[5] C. O. Pak, Photon Factory Activity Report No.4 A-11

[6] T. Obina, K. Haga, T. Honda, M. Tadano, T. Kasuga, M. Katoh, Y. Kobayashi, M. Satoh, and N. Nakamura, EPAC'98", Stockholm, (1998), 1726 